\title{
Representing Pairs of Orientations in the Plane
}

\author{
Magnus Herberthson ${ }^{1}$, Anders Brun ${ }^{2}$, and Hans Knutsson ${ }^{2}$ \\ ${ }^{1}$ Department of Mathematics, Linköping University, Sweden \\ ${ }^{2}$ Department of Biomedical Engineering, Linköping University, Sweden
}

\begin{abstract}
In this article we present a way of representing pairs of orientations in the plane. This is an extension of the familiar way of representing single orientations in the plane. Using this framework, pairs of lines can be added, scaled and averaged over in a sense which is to be described. In particular, single lines can be incorporated and handled simultaneously.
\end{abstract}

\section{Introduction}

Consider the two different rectangles in fig. 1 In each rectangle, two regions with different linear structure meet. If the dominant orientation is estimated, the (classical)
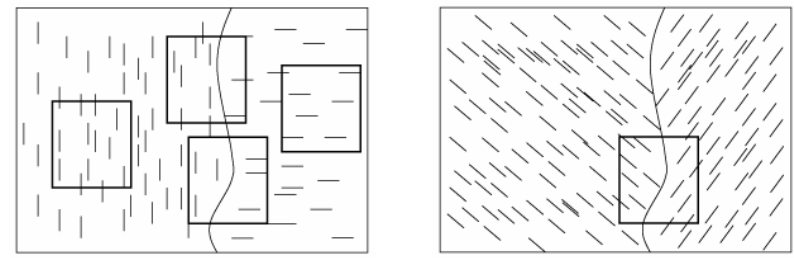

Fig. 1. When estimating a single orientation, it is hard to distinguish the border region in the rectangle to the left from the border region to the right. This can be remedied by allowing for pairs of orientations.

estimates formed near the border between the regions will be poor. The two middle 'windows' to the left and the window to the right will all give more or less the same information, namely that the average orientation is zero or isotropic. With the method presented here, these two cases can be distinguished.

\section{The Problem}

Let us consider the problem of adding, or making weighted averages of, pairs of indistinguishable orientations in the plane $\mathbf{P} \sim \mathbf{R}^{2}$, each direction being represented by a line through the origin. A pair of lines in $\mathbf{P}$, both containing the origin point, can be represented in several ways, for instance by the triple $(\hat{v}, \hat{u}, \alpha)$, where $\hat{v}$ and $\hat{u}$ are units vectors along the lines, and where $\alpha \in \mathbf{R}$ is a weight. It is noticed that $\pm \hat{v}$ and $\pm \hat{u}$ represent the same object, and any calculation must be invariant to these changes of 


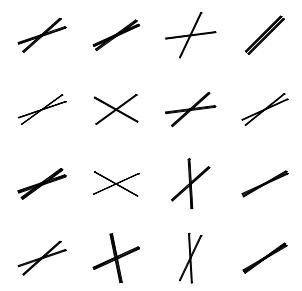

Fig. 2. A number of pairs of orientations in the plane. The thickness indicates strength and/or certainty. What is the dominating pair of orientations? How certain is it? How to you measure the 'spread'?

sign. Also, since the lines are indistinguishable, the representation should be invariant under the change $\hat{v} \longleftrightarrow \hat{u}$.

The situation is illustrated in figure 2, where we note that both lines in a pair of orientations have the same strength (or certainty). This is a condition which can be relaxed, i.e., the two orientations within a pair can indeed have different strengths, but this situation is most conveniently considered at a later stage. The problem is now the following:

Given a family of line pairs $\left\{\left(\hat{v}_{i}, \hat{u}_{i}, \alpha_{i}\right)\right\}_{i}$, how do we determine the (weighted) formal sum (or average) $\sum_{i=1}^{n}\left(\hat{v}_{i}, \hat{u}_{i}, \alpha_{i}\right)$ ?

\section{Sums of Single Lines}

In order to settle the notation, let us review the familiar corresponding process for a family of single lines, i.e., we consider, symbolically, $\sum_{i=1}^{n}\left(\hat{v}_{i}, \alpha_{i}\right)$. For a single line, the weight $\alpha$ can be encoded in $\bar{v}=\alpha \hat{v}$, so that we should consider the formal sum $\sum_{i=1}^{n} \bar{v}_{i}$ (or formal average $\frac{1}{n} \sum_{i=1}^{n} \bar{v}_{i}$ ), which again should be unaffected by any change(s) $\bar{v}_{i} \rightarrow-\bar{v}_{i}$. It is customary to form the sum of the outer products $A=\sum \bar{v}_{i} \bar{v}_{i}^{t}$, which gives a symmetric mapping $\mathbf{P} \rightarrow \mathbf{P}$ (or $\mathbf{R}^{n} \rightarrow \mathbf{R}^{n}$ if $v_{i} \in \mathbf{R}^{n}$ ). A can of course also be considered as a quadratic form. One then takes the largest eigenvalue and let the corresponding eigenline represent the average. See figure 3

Example 1. In standard coordinates, put $\hat{f}_{1}=\left(\begin{array}{l}1 \\ 0\end{array}\right), \hat{f}_{2}=\left(\begin{array}{l}0 \\ 1\end{array}\right), \hat{h}_{1}=\frac{1}{\sqrt{2}}\left(\begin{array}{l}1 \\ 1\end{array}\right)$, $\hat{h}_{2}=\frac{1}{\sqrt{2}}\left(\begin{array}{c}1 \\ -1\end{array}\right)$. Then $\hat{f}_{1} \hat{f}_{1}^{t}+\hat{f}_{2} \hat{f}_{2}^{t}=\left(\begin{array}{ll}1 & 0 \\ 0 & 1\end{array}\right)$. In both cases, the sum is isotropic, and no particular orientation can be distinguished, see figure 4 In addition, the information

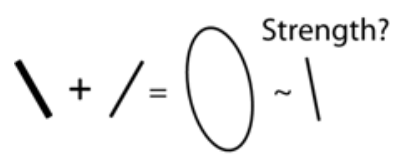

Fig. 3. The 'sum' of two lines is given by the major axis of the ellipse corresponding to the symmetric mapping formed from the lines. What is the strength? 


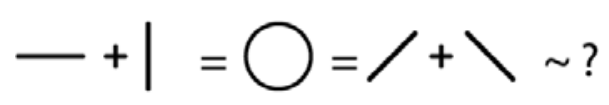

Fig. 4. Two different ways of adding up to a isotropic sum. No direction is singled out.

that the lines forming the sum were either horizontal/vertical or had slopes $\pm 45^{\circ}$ is completely lost. Cf. figures 9 and 10 . Remark: In the abstract index notation, [2], $A=$ $\sum \bar{v}_{i} \bar{v}_{i}^{t}$ would be written $A_{b}^{a}=\sum\left(v_{i}\right)^{a}\left(v_{i}\right)_{b}$, omitting the bars.

\section{Sums of Pair of Lines}

The triple $\left(\hat{v}_{i}, \hat{u}_{i}, \alpha_{i}\right)$ can be represented, non-uniquely, by the pair $\left(\bar{v}_{i}, \bar{u}_{i}\right) \sim\left(v^{a}, u^{a}\right)$, where $\alpha_{i}=\left|\bar{v}_{i}\right| \cdot\left|\bar{u}_{i}\right|$. Using this, we make the following definition.

Definition 1. Let a pair of orientations in the plane be given by the the pair $\left(v^{a}, u^{a}\right) \equiv$ $(\bar{v}, \bar{u})$ where $\pm \hat{v}, \pm \hat{u}$ are directed along the orientations and where $|\bar{v}| \cdot|\bar{u}|$ gives the strength. We then represent this pair of orientations by the symmetric tensor $v^{(a} u^{b)}:=$ $\frac{1}{2}\left(v^{a} u^{b}+v^{b} u^{a}\right)$.

In terms of column vectors, where $v^{a} \sim \bar{x}, u^{a} \sim \bar{y}, v^{(a} u^{b)}:=\frac{1}{2}\left(\bar{x} \bar{y}^{t}+\bar{y} \bar{x}^{t}\right)$, i.e. the symmetrized outer product. The primitive representations $v^{(a} u^{b)}$ specifies $v^{a}, u^{a}$ up to relative scaling and is not invariant under change of sign. To form outer products of such elements, they must live in a vector space. We provide $\mathbf{P}$ with the standard scalar product, and also introduce standard cartesian coordinates $(X, Y)$ so that $\hat{X}=\hat{f}_{1}$, $\hat{Y}=\hat{f}_{2}$.

Definition 2. We let $\mathbf{P}^{(a b)}$ denote the vector space of symmetric rank $\left(\begin{array}{l}2 \\ 0\end{array}\right)$ tensors over $\mathbf{P}$.

Elements in $\mathbf{P}^{(a b)}$ can be written either $v^{(a b)}$ or $v^{(a} u^{b)}$ where in the latter case it is understood that $v^{a} \in \mathbf{P}^{a}, u^{a} \in \mathbf{P}^{a}$. In terms of coordinates, elements in $\mathbf{P}^{(a b)}$ are represented by symmetric 2 by 2 matrices, in particular

Lemma 1. $\operatorname{dim}\left(\mathbf{P}^{(a b)}\right)=3$

Note however, that the set $\left\{v^{(a} u^{b)}, v^{a} \in \mathbf{P}^{a}, u^{a} \in \mathbf{P}^{a}\right\}$ does not constitute a vector space. As can easily be checked explicitly, cf. lemma 3, the symmetric 2 by 2 matrix $\hat{X}^{(a} \hat{X}^{b)}+\hat{Y}^{(a} \hat{Y}^{b)} \sim\left(\begin{array}{ll}1 & 0 \\ 0 & 0\end{array}\right)+\left(\begin{array}{ll}0 & 0 \\ 0 & 1\end{array}\right)=\left(\begin{array}{ll}1 & 0 \\ 0 & 1\end{array}\right)$ can not be written as $v^{(a} u^{b)}$ for any $v^{a}, u^{b}$. We now turn to the scalar product on $\mathbf{P}^{(a b)}$. This scalar product will be induced by the scalar product on $\mathbf{P}$, which we denote by $g_{a b}$. Thus $g_{a b}: \mathbf{P} \times \mathbf{P} \rightarrow \mathbf{R}$, so that $\bar{v} \cdot \bar{u}=g(\bar{v}, \bar{u})=g_{a b} v^{a} u^{b}=v^{a} u_{a}$. Again, the subscripts $a b$ in $g_{a b}$ indicates the covariant nature of the scalar product $g_{a b}$ as explained in [2].

Definition 3. The scalar product on $\mathbf{P}^{(a b)}: \mathbf{P}^{(a b)} \times \mathbf{P}^{(c d)} \rightarrow \mathbf{R}$ is given by $\left(v^{(a} u^{b)}, w^{(c} z^{d)}\right) \rightarrow g_{a c} g_{b d} v^{(a} u^{b)} w^{(c} z^{d)}=\frac{1}{2}\left(g_{a c} v^{a} w^{c} g_{b d} u^{b} z^{d}+g_{a c} v^{a} z^{c} g_{b d} u^{b} w^{d}\right)=$ $\frac{1}{2}((\bar{v} \cdot \bar{w})(\bar{u} \cdot \bar{z})+(\bar{v} \cdot \bar{z})(\bar{u} \cdot \bar{w}))$. 
In particular, the squared norm of the pair $\hat{v}^{(a} \hat{u}^{b)}$ is $\left\|\hat{v}^{(a} \hat{u}^{b)}\right\|^{2}=\frac{1}{2}\left[1+(\hat{v} \cdot \hat{u})^{2}\right]=$ $\frac{1}{2}\left[1+\cos ^{2} \phi\right]$, where $\phi$ is the angle between $\hat{v}$ and $\hat{u}$. One easily checks the following lemma.

Lemma 2. Let $v^{(a} u^{b)}$ and $w^{(c} z^{d)}$ be represented by the symmetric matrices $A$ and $B$. The scalar product is then simply given by $v^{(a} u^{b)} \cdot w^{(c} z^{d)}=\operatorname{tr}(A B)$.

It was noted above that the not every element of $\mathbf{P}^{(a b)}$ is of the primitive form $v^{(a} u^{b)}$. We therefore ask the question what elements in $\mathbf{P}^{(a b)}$ can be written as $v^{(a} u^{b)}$, with $v^{a} \in \mathbf{P}^{a}, u^{a} \in \mathbf{P}^{a}$ ? To illustrate this, we introduce suitable cartesian coordinates $x, y, z$ on $\mathbf{P}^{(a b)} \cong \mathbf{R}^{3}$.

In terms of standard coordinates, it is easy to see that an $\mathrm{ON}$-basis is given by

$$
\hat{e}_{1}=\frac{1}{\sqrt{2}}\left(\begin{array}{cc}
1 & 0 \\
0 & -1
\end{array}\right), \hat{e}_{2}=\frac{1}{\sqrt{2}}\left(\begin{array}{ll}
0 & 1 \\
1 & 0
\end{array}\right), \hat{e}_{3}=\frac{1}{\sqrt{2}}\left(\begin{array}{ll}
1 & 0 \\
0 & 1
\end{array}\right)
$$

We can now express each $v^{(a b)} \in \mathbf{P}^{(a b)}$ as $v^{(a b)}=x \hat{e}_{1}+y \hat{e}_{2}+z \hat{e}_{3}=\alpha^{1} \hat{e}_{1}+\alpha^{2} \hat{e}_{2}+$ $\alpha^{3} \hat{e}_{3}=\alpha^{j} \hat{e}_{j}$ or simply by the coordinates $\left[v^{(a b)}\right]=\left(\begin{array}{l}x \\ y \\ z\end{array}\right)$. One can easily check that $\hat{e}_{1}$ and $\hat{e}_{2}$, but not $\hat{e}_{3}$ can be decomposed. This is the content of the following lemma.

Lemma 3. Suppose $\left[w^{(a b)}\right]=\left(\begin{array}{l}x \\ y \\ z\end{array}\right)$ is given. Then $w^{(a b)} \in \mathbf{P}^{(a b)}$ is decomposable as $w^{(a b)}=v^{(a} u^{b)}$ for some $v^{a}, u^{a} \in V^{a}$ if and only if $z^{2} \leq x^{2}+y^{2}$.

The proof is fairly straight forward, using the ansatz $\hat{v}=\left(\begin{array}{c}\cos \phi \\ \sin \phi\end{array}\right), \hat{u}=\left(\begin{array}{c}\cos \gamma \\ \sin \gamma\end{array}\right)$.

\subsection{Illustrations of Pair of Orientations in $\mathrm{P}^{(a b)}$}

We have introduced cartesian coordinates $x, y, z$ in $\mathbf{P}^{(a b)}$, and know that that decomposable primitive objects corresponds to vectors whose coordinates satisfies $z^{2} \leq x^{2}+y^{2}$. We also have the ambiguity that $v^{(a b)}$ and $-v^{(a b)}$ represent the same pair of orientations (and strength) in the plane $\mathbf{P}$.

Apart from the strength, each pair of orientations can be represented by the symmetrized outer product of $\hat{v}_{\phi}$ and $\hat{u}_{\gamma}$ where $\phi$ and $\gamma$ indicates the angle between the unit vector in question and a reference direction, e.g. the positive $x$-axis. Since both unit vectors are interchangeable, and since $\hat{v}_{\phi}$ and $\hat{v}_{\phi+\pi}$ represent the same orientation, many different pairs of angles $(\phi, \gamma)$ will represent the same pair of orientation. This is illustrated in figure 5 where the gray triangle gives an example of a region containing all possible values of $\hat{v}_{\phi}^{(a} \hat{u}_{\gamma}^{b)}$. In this triangle, each object $\hat{v}_{\phi}^{(a} \hat{u}_{\gamma}^{b)}$ is represented by a single point, except for the boundary points, which are to be identified in a certain way. For instance, in figure 5, the three small squares all represent the same pair of orientations. Similarly, the two small circles are to identified. As can be anticipated, the topology of this triangle with appropriate boundary points identified is non-trivial. (In fact, the topology is the Moebuis band.) 


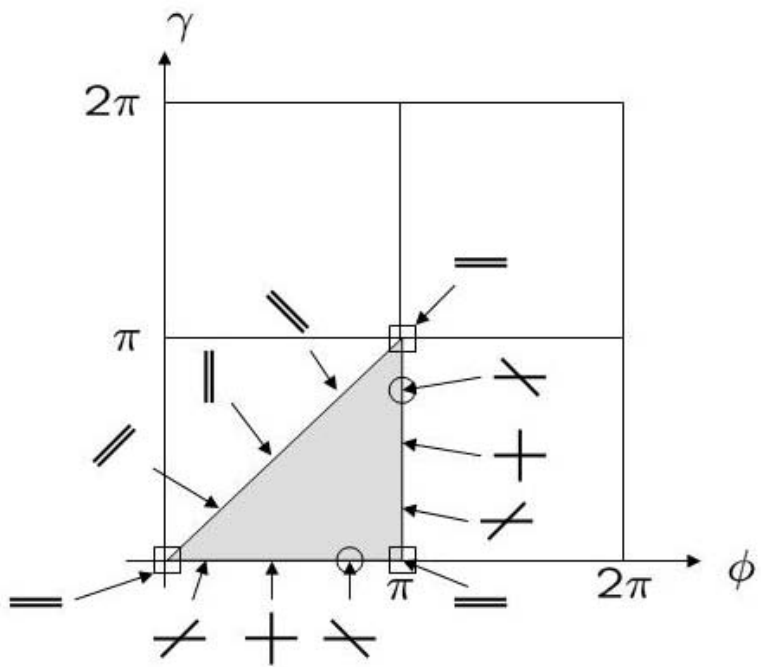

Fig. 5. The gray triangle is an example of a region where each element $v^{(a b)} \in \mathbf{P}^{(a b)}$ i uniquely represented by one point, except for the boundary of the triangle where certain points are to be identified. This identification gives a non-trivial topology.

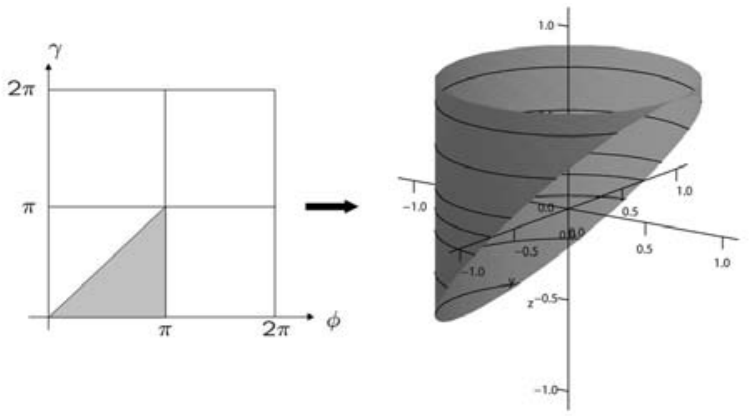

Fig. 6. The triangle is mapped in an almost one-to-one fashion to the surface to the right. Antipodal points on the ellipse are to be identified.

Each element $\hat{v}_{\phi}^{(a} \hat{u}_{\gamma}^{b)}$ is mapped in to $\mathbf{P}^{(a b)}$, and consequently, the set $M=\left\{\hat{v}_{\phi}^{(a} \hat{u}_{\gamma}^{b)} \mid\right.$ $0 \leq \phi \leq 2 \pi, 0 \leq \gamma \leq 2 \pi\}$ is represented by a surface in $\mathbf{P}^{(a b)}$. In terms of the coordinates used in lemma 3 , the surface $\mathrm{M}$ occupies the cylinder $\left\{(x, y, z) \mid x^{2}+y^{2}=\right.$ $1,-1 \leq z \leq 1\}$. However, the image of $M$ is not injective, since the elements $\hat{v}_{\phi}^{(a} \hat{u}_{\gamma}^{b)}$ and $\hat{v}_{\phi}^{(a} \hat{u}_{\gamma+\pi}^{b)}$ represent the same pair of orientations, but differ by a sign when represented in $\mathbf{P}^{(a b)}$, antipodal points in $\mathbf{P}^{(a b)}$ must be identified. In order to get a (almost) one-to-one mapping between elements in a part of $M$ and a surface in $\mathbf{P}^{(a b)}$, we look at figure 6. Apart from the boundary points of the indicated triangle, each element represents a pair of orientation uniquely. The triangle is mapped to the cylindrical surface to the right, where now only antipodal points on the shown ellipse are to be identified. 


\subsection{Sums or Averages of Pairs of Orientations}

To each pair of orientations in the plane $\mathbf{P}$, we have the associated element $v^{(a} u^{b)} \in$ $\mathbf{P}^{(a b)}$. This representation is not invariant under the inversion $v \rightarrow-v$ or $u \rightarrow-u$, which is reflected by the property that antipodal elements in $\mathbf{P}^{(a b)}$ are to be identified. To handle this sign ambiguity, we proceed as in the single line case, i.e., we note that the outer product of $v^{(a} u^{b)} v_{(c} u_{d)}$ is invariant under $v \rightarrow-v$ or $u \rightarrow-u$. Thus, repeating the steps for the single line case, the procedure is as follows.

Suppose that we are given $n$ elements $v_{i}^{(a} u_{i}^{b)} \in \mathbf{P}^{(a b)}$. To calculate their formal 'sum', we form the sum of the tensor products

$$
\sum_{i=1}^{n} v_{i}^{(a} u_{i}^{b)}\left(v_{i}\right)_{(c}\left(u_{i}\right)_{d)}
$$

This gives a symmetric mapping $\mathbf{P}^{(a b)} \rightarrow \mathbf{P}^{(a b)}$. We then let the eigenvectors corresponding to the largest eigenvalue of this mapping correspond to the 'sum', or dominating pair of orientations, if these eigenvectors (in $\mathbf{P}^{(a b)}$ ) are decomposable.

Again we remark that the sum should be regarded is the symmetric mapping as a whole, and that if the eigenvalues of this mapping are of the same order, it may be highly missleading to focus on the largest eigenvalue.

In this case, we also have the complication that the eigenvectors in $\mathbf{P}^{(a b)}$ corresponding to the largest eigenvalue, may not be decomposable as a pair of lines in $\mathbf{P}$. However, this is not a real problem as this situation corresponds to an isotropic situation, where the two lines are replaced by a ellipsoid (or circle).

Example 2. In figure 7 we have started with four pairs of lines in the plane, drawn to the left. By representing them in $\mathbf{P}^{(a b)}$, forming the outer products and summing, we get a symmetric mapping, whose principal ellipsoid is drawn to the right. The pair of lines corresponding to the eigenvector with the largest eigenvalue is also shown.

\subsection{Some Properties of Pair Orientation Averages}

In this section we mention some of the properties of the presented representation.

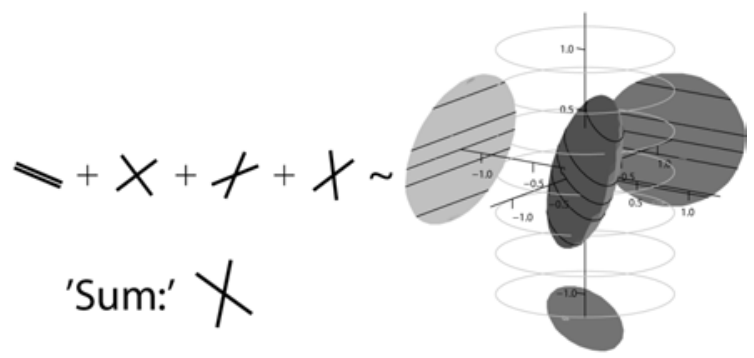

Fig. 7. The sum of four pairs of lines. The resulting ellipsoid is shown to the left, together with its projections on the coordinate planes. 
Sums Containing a Common Orientation. A natural situation is to consider sums of pair of orientations $\sum_{i} v^{(a} u_{i}{ }^{b)}$, i.e., we have an orientation $v^{a}$ which is common to all pairs. The natural question is to ask then whether the resulting (dominant) pair of orientations always will contain the orientation represented by $\hat{v}$ or not. For consistency reasons, we expect the answer to be yes, and that this is the case is content of the following theorem.

Theorem 1. Suppose that we are given $n$ elements $v^{(a} u_{i}^{b)}$, so that all elements contain a common orientation (given by $v$ ). If we form outer products of these elements regarded as vectors in $\mathbf{P}^{(a b)}$, and sum them to a symmetric mapping $\mathbf{P}^{(a b)} \rightarrow \mathbf{P}^{(a b)}$, then any eigenvector corresponding to the largest eigenvalue will contain $v^{a}$, i.e., it can be written $v^{(a} u^{b)}$ for some $u^{b} \in \mathbf{P}^{b}$.

This is illustrated in figure 8 , where all pairs contain a common direction. The corresponding ellipsoid lies in a plane, which means that one eigenvalue is zero. Again, the 'dominating' pair is also shown.

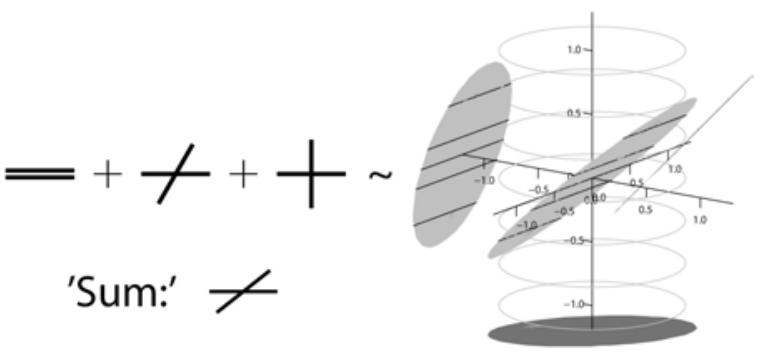

Fig. 8. The sum of three pairs of lines. This time all pairs have one common orientation. As a result, the corresponding ellipsoid lies in a plane, i.e. one eigenvalue is 0 .

Before we start the proof of theorem 1 we introduce a notation and a useful lemma.

Definition 4. Suppose that $v \in \mathbf{P}$ is given. $v_{\perp} \in \mathbf{P}$ is then defined as the vector obtained by rotating $v \pi / 2$ counter-clockwise. Furthermore, by $v_{\perp}^{(a b)} \in \mathbf{P}^{(a b)}$ we mean $v_{\perp}^{(a b)}=v_{\perp}^{(a} v_{\perp}^{b)}$

By construction, $v$ and $v_{\perp}$ are orthogonal, i.e., $v_{\perp}^{a} v_{a}=0$. As regards $v_{\perp}^{(a b)}$, we have the following lemma.

Lemma 4. Let $v^{a} \in V^{a}$ be given. Then $v_{\perp}^{(a b)} u_{(a b)}=0 \Leftrightarrow u^{(a b)}=v^{(a} u^{b)}$ for some $u^{a} \in \mathbf{P}^{a}$.

\section{Proof of lemma 4}

$\Leftarrow$ : Suppose $u^{(a b)}=v^{(a} u^{b)}$ for some $u^{a} \in \mathbf{P}^{a}$. Then $v_{\perp}^{(a b)} u_{(a b)}=v_{\perp}^{(a} v_{\perp}^{b)} v_{(a} u_{b)}=0$.

$\Rightarrow$ : It is easy to see that for a given (non-zero) $v^{a} \in \mathbf{P}^{a}$, the set $\Pi=\left\{v^{(a} u^{b)}: u^{a} \in\right.$ $\left.V^{a}\right\}$ is a two-dimensional vector space. Also, $\Pi$ lies in the orthogonal complement to $v_{\perp}^{(a b)}:\left[v_{\perp}^{(a b)}\right]^{\perp}$. But since $v_{\perp}^{(a b)}$ is a non-zero vector in the three-dimensional vector space $\mathbf{P}^{(a b)}$, its orthogonal complement $\left[v_{\perp}^{(a b)}\right]^{\perp}$ is two-dimensional, i.e., it is $\Pi$. 


\section{Proof of theorem 1}

Put $\Pi=\left[v_{\perp}^{(a b)}\right]^{\perp}$. All elements $v^{(a} u_{i}{ }^{b)}$ lie in $\Pi$. Therefore, to the (positive semidefinite) symmetric mapping formed by the sum of the outer products of these elements, all eigenvectors corresponding to nonzero eigenvalues must lie in $\Pi$. This is perhaps most easily seen as a consequence of the fact that $v_{\perp}^{(a b)}$ is an eigenvector with eigenvalue 0 , and that eigenvectors corresponding to different eigenvalues are orthogonal.

Representing Single Orientations. As we saw in example 1 and figure 4 , the sum of two lines may be totally isotropic, which in particular means that all information of the

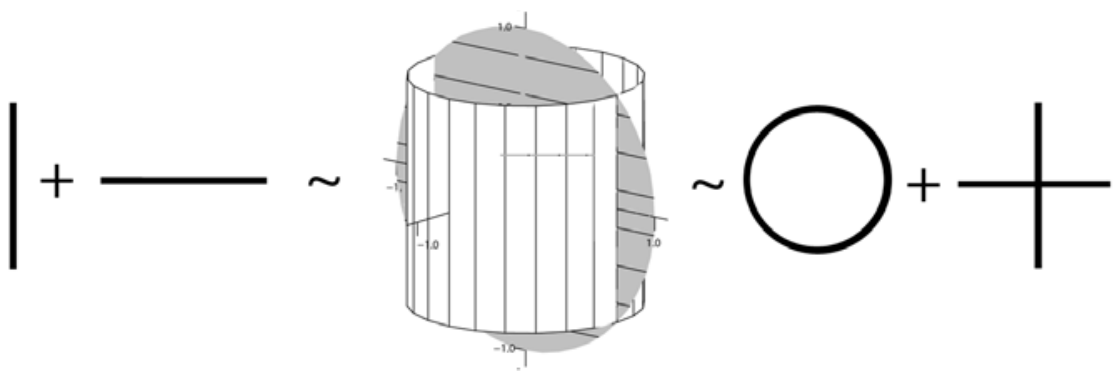

Fig. 9. The two lines to the left are each represented by pair of identical orientations. Their sum is represented by the disk in the figure, which can equally well be interpreted as the sum of the two objects to the right. Cf. figure 10

individual lines are lost. Clearly, it could be advantageous to find the isotropic sum, but still be able to distinguish between the two cases (and others) in figure 4 This can be done by representing the orientation of one single line as a pair of parallel coinciding lines in the manner described in this paper. In both cases, there is a common eigenvector which is vertical in the figures 9 and 10 . This eigenvector is not decomposable, i.e. can not be written as $v^{(a} u^{b)}$, and corresponds to the isotropic part to the right. However, the eigenvectors orthogonal to the common vertical eigenvector differs in the cases displayed in the figures 9 and 10 Each of these vectors is decomposable and corresponds to pairs of orthogonal lines, as shown in the figures.

\subsection{Pair of Lines with Different Strength}

Although explicitly stated earlier that the orientations we have represented should be indistinguishable, this statement can be slightly modified. Uptil now, we have not assigned any individual strength to $v^{a}$ or $u^{a}$ in the composite object $v^{(a} u^{b)}$, i.e., from the composite object, the constituent parts are only determined up to a scaling. However, the crucial property is rather that when summing pairs of orientations, $v^{(a} u^{b)}$ with $w^{(a} z^{b)}$ say, there should not be any rule connecting $v^{a}$ with either $w^{a}$ or $z^{a}$. Apart from that, one could very well consider cases where the lines/orientations have different strength. This is modeled in such a way that the orientations given by $v^{a}$ and $u^{a}$, where the line given by $v^{a}$ is 'stronger' than the one given by $u^{a}$ is modeled as a sum of $v^{(a} v^{b)}$ and $v^{(a} u^{b)}$. 


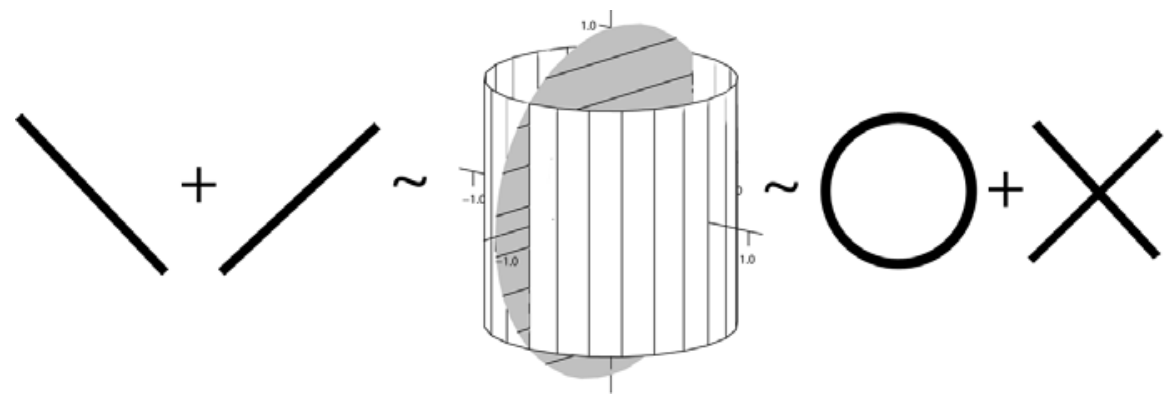

Fig. 10. The two lines to the left are each represented by pair of identical orientations. Their sum is represented by the disk in the figure, which can equally well be interpreted as the sum of the two objects to the right. Compared to figure 9 both disks have a common vertical (eigen) vector. This corresponds to the common isotropic part. The horisontal vectors contained in the discs in this and the previous figure differ, which shows up by the different orientations of the orthogonal line pairs.

\subsection{Generalizations}

The concept presented here has obvious generalisations . For instance, one might consider pairs of lines in a vector space $V$ with $\operatorname{dim}(V)=3$ instead of 2 . The same construction is useful, i.e., one consider elements $v^{(a} u^{b)} \in V^{(a b)}$ where $v, u \in V$. Most earlier calculations have obvious counterpart, the most important difference is perhaps the correspondence to lemma 3 This time, we can regard elements in $V^{(a b)}$ as symmetric $3 \times 3$ matrices. However, elements decomposable as $v^{(a} u^{b)}$ will, among other conditions, correspond to a surface (rather than a volume) in $V^{(a b)}$, since the corresponding symmetric matrices have rank 2 . On the other hand, one can equally well consider three orientations in a three-dimensional space, i.e., elements of the type $v^{(a} u^{b} w^{c)}$. These elements lie in vector space of dimension 10. To proceed, one can consider $n$ lines (through the origin) in a $m$-dimensional vector space $V$, by forming the symmetrized outher products $v_{1}^{(a} v_{2}^{b} \ldots v_{n}^{c)}$.

\subsection{Example/Applications}

Let us return to the situation in figure 1 There we considered the problem of estimating the dominating orientation of linear structures. Handled in the traditional way, these estimates normally fail to describe the situation satisfactory near 'borders' i.e., where two areas with different orientations meet. See also figures 49 and 10 Using the approach desribed in this work, the situation becomes different. This is illustrated in figure 11 where, to the left, two regions with different orientations meet. Using a kernel with $5 \times 5$ elements, averages of neighbouring orientations are formed, and these averages are then presented as pairs of lines. Away from the border these pairs are dominated by a single orientations, while in the transit region, the averages show up as crosses, reflecting both ingoing orientations. Among others, it is possible to discriminate between the different sitations described in figure 4 . Further postprocessing and presentation is of course application dependent. 

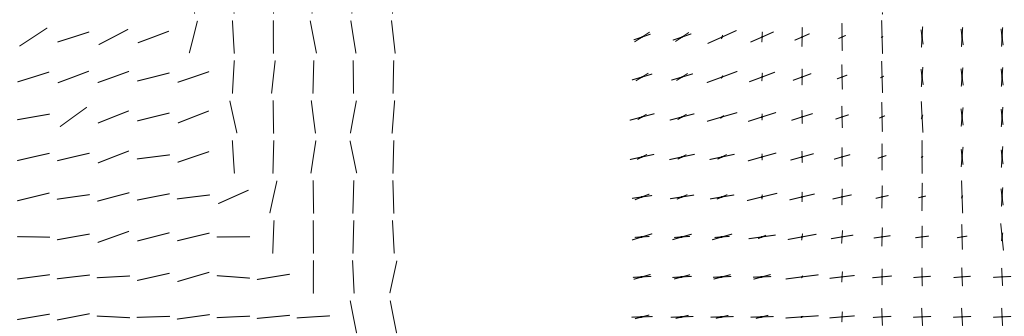

Fig. 11. To the left: two regins with different orientations meet. To the right: Estimates of the local orientations are given. By using the framework presented in this work, the estimates near the border between the regions are pairs of lines rather than isotropic circles or near isotropic ellipses.

\section{References}

1. Knutsson, H.: Representing Local Structure Using Tensors. The 6th Scandinavian Conference on Image Analysis, Oulu, Finland (1989)

2. Wald, R.M.: General Relativity, University of Chicago Press (1984) 